\title{
The abundance of the Lyme disease pathogen Borrelia afzelii declines over time in the tick vector Ixodes ricinus
}

\author{
Maxime Jacquet, Dolores Genné, Alessandro Belli, Elodie Maluenda, Anouk Sarr and Maarten J. Voordouw*
}

\begin{abstract}
Background: The population dynamics of vector-borne pathogens inside the arthropod vector can have important consequences for vector-to-host transmission. Tick-borne spirochete bacteria of the Borrelia burgdorferi (sensu lato) species complex cause Lyme borreliosis in humans and spend long periods of time (>12 months) in their Ixodes tick vectors. To date, few studies have investigated the dynamics of Borrelia spirochete populations in unfed Ixodes nymphal ticks.
\end{abstract}

Methods: Larval ticks from our laboratory colony of I. ricinus were experimentally infected with B. afzelii, and killed at 1 month and 4 months after the larva-to-nymph moult. The spirochete load was also compared between engorged larval ticks and unfed nymphs (from the same cohort) and between unfed nymphs and unfed adult ticks (from the same cohort). The spirochete load of B. afzelii in each tick was estimated using qPCR.

Results: The mean spirochete load in the 1-month-old nymphs ( 14,000 spirochetes) was seven times higher than the 4-month-old nymphs ( 2000 spirochetes). Thus, the nymphal spirochete load declined by $80 \%$ over a period of 3 months. An engorged larval tick acquired 100 spirochetes, and this population was 20 times larger in a young, unfed nymph. The spirochete load also appeared to decline in adult ticks. Comparison between wild and laboratory populations found that lab ticks were more susceptible to acquiring $B$. afzelii.

Conclusion: The spirochete load of B. afzelii declines dramatically over time in domesticated I. ricinus nymphs under laboratory conditions. Future studies should investigate whether temporal declines in spirochete load occur in wild Ixodes ticks under natural conditions and whether these declines influence the tick-to-host transmission of Borrelia.

Keywords: Borrelia afzelii, Host-to-tick transmission, Ixodes ricinus, Lyme borreliosis, Pathogen burden, Spirochete load, Tick-borne disease, Vector-borne disease

\section{Background}

Vector-borne pathogens reproduce, develop, and migrate inside their arthropod vector. The population dynamics of vector-borne pathogens inside their arthropod vectors plays a critical role in the transmission of vector-borne diseases. The number of pathogens inoculated by the arthropod vector into the vertebrate host is one of the key predictors of vector-to-host transmission success [1-3], and this variable often depends on the duration of vector feeding, which gives the pathogen population time to replicate and migrate to the relevant tissues $[4,5]$. For

\footnotetext{
* Correspondence: maarten.voordouw@unine.ch Laboratory of Ecology and Evolution of Parasites, Institute of Biology,

University of Neuchâtel, Neuchâtel, Switzerland
}

many arboviruses, the speed at which mosquito vectors become infectious to their vertebrate hosts depends on the population growth rate of the virus inside the vector, which depends on extrinsic environmental factors such as temperature $[2,6,7]$. Studies of tick-borne pathogens that establish multiple-strain infections have found that the abundance of a particular strain in the arthropod vector is an important predictor of strain-specific transmission and fitness [8-10]. In summary, the study of the factors that influence the pathogen population size inside the arthropod vector may give us a better understanding of the epidemiology of vector-borne diseases [11].

Borrelia burgdorferi (sensu lato) (s.l.) is a species complex of spirochete bacteria that cause Lyme borreliosis 
(LB) in humans, and that are transmitted by Ixodes ticks [12-14]. Larval ticks acquire Borrelia spirochetes from infected vertebrate reservoir hosts and moult into infected nymphs, which transmit the pathogen to new hosts. The larval and nymphal blood meals occur in consecutive summers, and the Borrelia spirochetes spend 1 year or more in the immature tick [15-17]. How Borrelia spirochetes persist inside unfed Ixodes nymphs is currently unknown [18], but there is a lot of interest in identifying the underlying genes [18-24]. Studies that have characterised Borrelia population dynamics in Ixodes nymphs have mostly focused on the short duration of attachment to the vertebrate host ( $<7$ days) and the resulting pathogen transmission [25-31]. In contrast, there are not many studies that have investigated spirochete population dynamics during the long sojourn inside unfed nymphal ticks. Early microscope studies on B. burgdorferi (sensu stricto) (s.s.) in I. scapularis found that the spirochete abundance in the nymphal tick following the larva-to-nymph moult was low ( 300 spirochetes) $[26,32]$, suggesting that it was constant over time. In contrast, a recent study on the European LB pathogen, B. afzelii, in its tick vector, I. ricinus, observed that the spirochete population in the nymphal tick following the larva-to-nymph moult was high (>10,000 spirochetes) and that it declined dramatically (by almost 90\%) over a period of 6 months [33]. This observed decline in spirochete load was a serendipitous discovery, and the study was not designed to answer that particular question [33]. The purpose of the present study is therefore to test whether the spirochete load of $B$. afzelii changes over time in unfed $I$. ricinus nymphs. The spirochete load was also investigated in engorged larval ticks and unfed adult ticks to obtain a better understanding of how the population size of $B$. afzelii inside $I$. ricinus changes over the life-cycle of the tick vector.

\section{Methods}

\section{Strains of Borrelia afzelii}

Borrelia afzelii isolates E61 and NE4049 were used in this study. These isolates have ID numbers 1888 and 1887 in the Borrelia MLST database, respectively. E61 was originally isolated from a human patient in Austria whereas NE4049 was isolated from an $I$. ricinus tick in Neuchâtel, Switzerland. Isolate E61 has multi-locus sequence type (MLST) ST75 and ospC major group (oMG) A3, whereas isolate NE4049 has ST679 and oMG A10. For simplicity and as we have done elsewhere, these two isolates will hereafter be referred to as B. afzelii ospC strains A3 and A10 [3335]. We have previously characterised the co-feeding and systemic transmission phenotypes of these two $o s p C$ strains over the acute and chronic phase of the infection [33-36].
Creation of $B$. afzelii-infected laboratory I. ricinus nymphs killed at 1 and 4 months post-moult

Female Mus musculus Balb/cByJ mice were experimentally infected with either B. afzelii ospC strain A3 or strain A10 via nymphal tick bite. The details of this infection experiment have been described elsewhere [33, 34, 36]. There were 10 and 13 mice that became systemically infected with strain A3 and strain A10, respectively. The mice were infested with larval $I$. ricinus ticks on five separate occasions at 2, 34, 66, 94, and 128 days post-infection (PI). For each infestation, 50-100 larvae were placed on each mouse. All ticks came from the pathogen-free, laboratory colony of I. ricinus at the University of Neuchâtel. Engorged larval ticks were placed in individual tubes and were allowed to moult into nymphs $[33,34,36]$. Larval and nymphal ticks were kept under controlled laboratory conditions: the temperature was $22{ }^{\circ} \mathrm{C}$ and the relative humidity was $>80 \%$. The present study only concerns the larval ticks from the last three infestations $(66,94$, and 128 days PI). For each of the 69 combinations of mouse $(n=23$ mice) and infestation $(66,94$, and 128 days PI), we randomly selected a maximum of 10 nymphs and froze them at $-20{ }^{\circ} \mathrm{C}$ at 1 and 4 months after the larva-to-nymph moult.

\section{Spirochete load of $B$. afzelii in engorged larvae and flat nymphs}

To determine how the abundance of $B$. afzelii changes over the course of the life-cycle of $I$. ricinus, the spirochete loads were compared between engorged larval ticks and flat nymphs. Each of 10 female Balb/cByJ mice was experimentally infected with $B$. afzelii ospC strain A10 via nymphal tick bite. Four weeks after the nymphal challenge, $B$. afzelii infection in the mice was confirmed with a Borrelia-specific qPCR assay on an ear tissue sample and a commercial ELISA to detect Borrelia-specific IgG antibodies. Five weeks after the nymphal challenge, each mouse was challenged with $\sim 100$ pathogen-free xenodiagnostic larvae from our laboratory colony of $I$. ricinus. Blood-engorged larvae were placed in individual Eppendorf tubes, and 5 engorged larvae were randomly selected for each mouse and frozen at $-20{ }^{\circ} \mathrm{C}$ within $48 \mathrm{~h}$ of drop-off. The remaining engorged larval ticks were allowed to moult into nymphs under controlled laboratory conditions. At 4 weeks after the larva-tonymph moult, 10 live nymphs were randomly selected for each mouse and frozen at $-20^{\circ} \mathrm{C}$.

\section{Spirochete load of $B$. afzelii in flat nymphs and flat adult ticks}

To determine how the abundance of $B$. afzelii changes over the course of the life-cycle of I. ricinus, the spirochete loads were compared between flat nymphs and flat adult ticks. Each of 7 mice infected via nymphal tick bite with B. afzelii ospC strain A10 was infested with larval 
ticks from our laboratory colony of $I$. ricinus at 30 days post-infection. The engorged larval ticks were allowed to moult into nymphs. To test for the prevalence of $B$. afzelii infection, 5 nymphs were randomly sampled from each mouse and frozen at $-20{ }^{\circ} \mathrm{C}$ at 4 weeks after the larva-to-nymph moult. At $\sim 170$ days after the larval blood meal and $\sim 90$ days after the larva-to-nymph moult, 100 of these nymphal ticks were fed on 20 pathogen-free, female Balb/cByJ mice (5 nymphs/ mouse). Nymphs were placed in plastic capsules that were attached to the backs of the mice, as we have described previously [33]. The engorged nymphal ticks were kept in individual Eppendorf tubes under standard conditions and were allowed to moult into adult ticks. The adult ticks were killed on a date that corresponded to the following times in the tick life-cycle: 198 days (28 weeks) after the nymph-to-adult moult, 271 days (39 weeks) after the nymphal blood meal, and 362 days (52 weeks) after the larva-to-nymph moult.

\section{Creation of $B$. afzelii-infected wild $I$. ricinus nymphs frozen at 1 month post-moult}

Wild I. ricinus larval ticks were collected from a field site near Neuchâtel, Switzerland in the summer of 2014. Ten female Balb/cByJ mice were experimentally infected with isolate NE4049 via nymphal tick bite. At 5 weeks PI, infection of the mice was confirmed using a commercial Lyme disease ELISA. At 6 weeks PI, batches of 100 wild larvae were allowed to feed on the experimentally infected mice. Engorged larval ticks were collected and stored under controlled laboratory conditions as above. For each of the 10 mice, 10 nymphs were randomly selected and frozen at $-20{ }^{\circ} \mathrm{C}$ at 1 month after the larva-to-nymph moult.

\section{DNA extraction of nymphal ticks and qPCR to determine B. afzelii infection}

Total DNA was extracted from individual ticks using a TissueLyser II and DNeasy 96 Blood \& Tissue kit well plates (Qiagen, Hilden, Germany). The DNA extraction protocol was described in a previous study [33]. A quantitative PCR amplifying a 132 bp fragment of the flagellin gene [37] was used to detect and quantify Borrelia DNA. The qPCR protocol was described in a previous study [33]. For the 1-month-old ticks in the first experiment, we conducted three replicate qPCR runs for each tick. The repeatability of the $\log 10$-transformed spirochete load estimated by these three replicate runs was very high $(r=0.972)$ [33]. We therefore ran single qPCR runs for the other ticks.

\section{Statistical analysis}

All statistical analyses were done in $\mathrm{R}$ version 3.1.0. [38]. The datasets generated and analysed in the present study are available in Additional file 1.

\section{Paired data for 1-month-old nymphs and 4-month-old nymphs}

In the first experiment, paired data were obtained on 1-month-old nymphs and 4-month-old nymphs for 43 of the 69 possible combinations of mouse $(n=$ 23 mice) and infestation (66, 94, and 128 days PI). The data are paired because the 1-month-old nymphs and the 4-month-old nymphs fed as larvae on the same mouse during the same infestation. The only difference between these two groups of nymphs is that they were killed at different times following the larva-to-nymph moult.

\section{Definition of B. afzelii infection status for nymphal ticks}

The nymphs were considered as infected with $B$. afzelii if the nymphal spirochete load estimated by the $\mathrm{qPCR}$ was $>1$. For the first experiment, the proportions of infected ticks for the 1-month-old nymphs and the 4month-old nymphs were calculated for each of the 43 available combinations of mouse and infestation.

\section{Spirochete loads of B. afzelii-infected nymphal ticks}

The total spirochete load for each nymph was calculated by adjusting the spirochete estimate for $5 \mu$ l of DNA template of the $\mathrm{qPCR}$ reaction to the volume of the DNA extraction $(65 \mu \mathrm{l})$. As gene copy data from qPCR assays follow an exponential distribution, the spirochete loads were $\log 10$-transformed to improve their fit to the normal distribution. In the first experiment, the spirochete load of each 1-month-old nymph was the average of the three replicate qPCR runs (negative runs were excluded). The spirochete load of each 4-month-old nymph and each tick in the other experiments was the estimate from the single qPCR run. For each of the 43 infestations in the first experiment, the mean nymphal spirochete load was calculated separately for the 1-month-old nymphs and the 4-month-old nymphs using the subset of infected ticks (negative ticks were excluded).

\section{Effect of time on the nymphal spirochete load of B. afzelii}

Means and standard errors of the spirochete load were calculated on the $\log 10$-transformed scale before being back-transformed to the original scale. For the first experiment, a paired $t$-test was used to compare the mean proportion of infected ticks and the mean $\log 10$ transformed spirochete load between the 1-month-old nymphs and the 4-month-old nymphs. For the first experiment, Pearson's correlation was used to test whether the proportion of infected ticks and the $\log 10$ transformed spirochete load were correlated between the 1-month-old nymphs and the 4-month-old nymphs. 


\section{Results}

Effect of nymphal age on the probability of detecting $B$. afzelii in nymphal ticks

The mean proportion of infected nymphs was similar between the 1-month-old nymphs $(61.8 \%=259 / 419)$ and the 4-month-old nymphs $(64.3 \%=173 / 269)$ and the difference was not statistically significant $(t=0.695, d f=42$, $P=0.491)$.

\section{Effect of nymphal age on the spirochete load of infected nymphs}

The mean spirochete load of the 1-month-old nymphs ( $n=43$, mean: $14,094,95 \%$ confidence interval (CI): 13,508-14,705 spirochetes per nymph) was 7.05 times larger than that of the 4-month-old nymphs $(n=42$, mean: 1999, 95\% CI: 1900-2103 spirochetes per nymph) and this difference was highly significant $(t=12.768$, $d f=41, P<0.0001)$. The mean spirochete load of the 1-month-old nymphs was higher than that of the 4month-old nymphs for 40 of the 42 pairs for which there were data.

For strain A3, the mean spirochete load of the 1month-old nymphs $(n=22$, mean: 8880, 95\% CI: 8169-9654 spirochetes per nymph) was 5.8 times larger than the 4-month-old nymphs $(n=22$, mean:
1525, 95\% CI: 1367-1700 spirochetes per nymph) and this difference was significant $(t=7.804, d f=21, P<$ 0.0001; Fig. 1). For strain A3, the flat nymphs lost an average of 81.7 spirochetes per day. For strain A10, the mean spirochete load of the 1-month-old nymphs $(n=21$, mean: 22,865, 95\% CI: 21,444-24,380 spirochetes per nymph) was 8.5 times larger than that of the 4-month-old nymphs $(n=20$, mean: 2693, 95\% CI: 2459-2949 spirochetes per nymph) and this difference was significant $(t=10.825, d f=19, P<0.0001$; Fig. 1). For strain A10, the flat nymphs lost an average of 208.3 spirochetes per day.

\section{Correlations in phenotype between young and old nymphs}

The proportion of infected nymphs was not correlated between the 1-month-old nymphs and the 4-month-old nymphs $(r=0.161, t=1.046, d f=41, P=0.302)$. In contrast, the mean log10-transformed spirochete load was highly correlated between the 1-month-old nymphs and the 4-month-old nymphs $(r=0.510, t=$ $3.745, d f=40, P<0.001)$. After removing the 10 pairs for which less than five 4-month-old nymphs were recovered, the magnitude of the correlation of the log10-transformed spirochete load increased ( $r=$ 0.580, $t=3.966, d f=31, P<0.001$; Fig. 2).

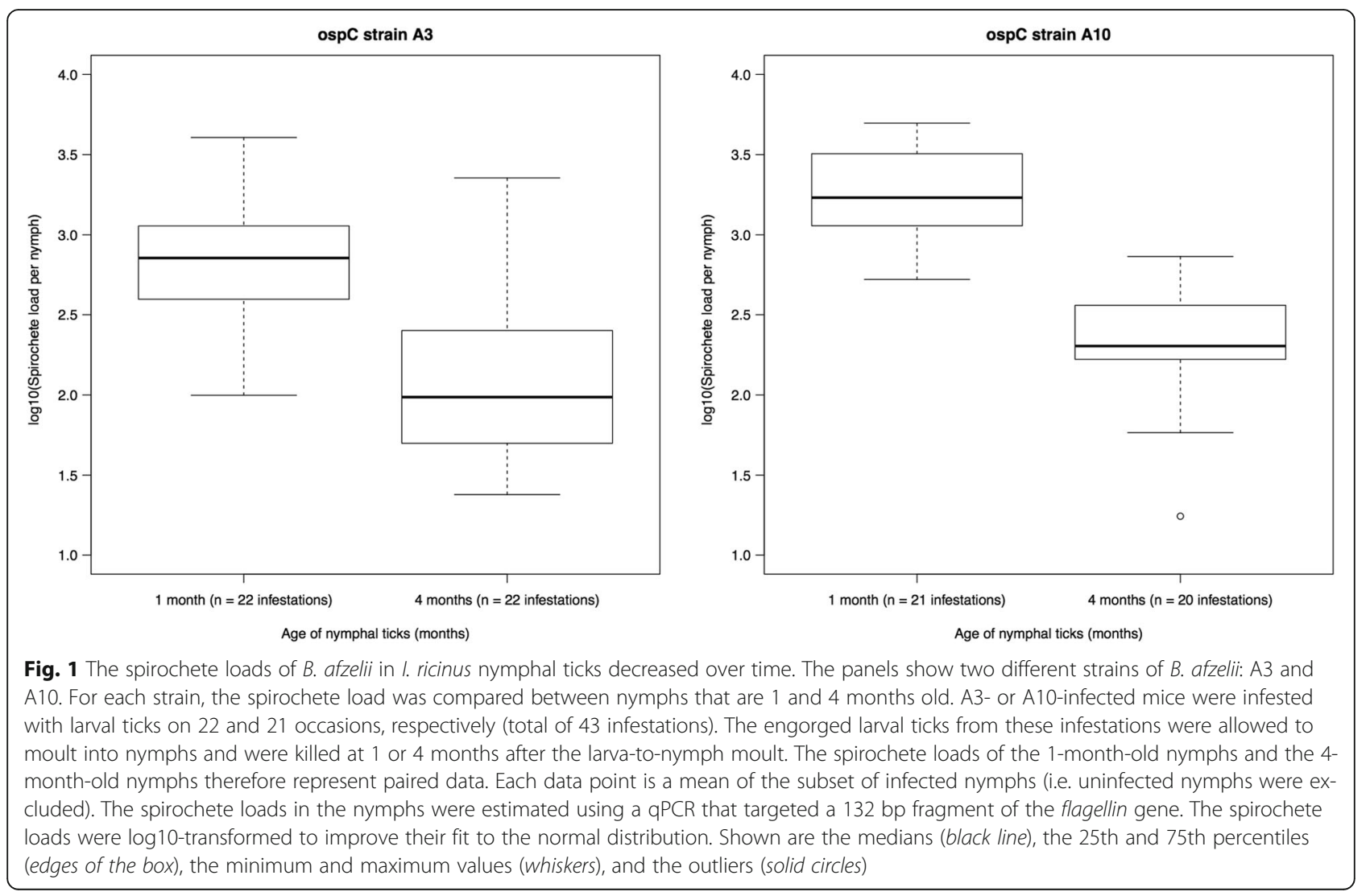




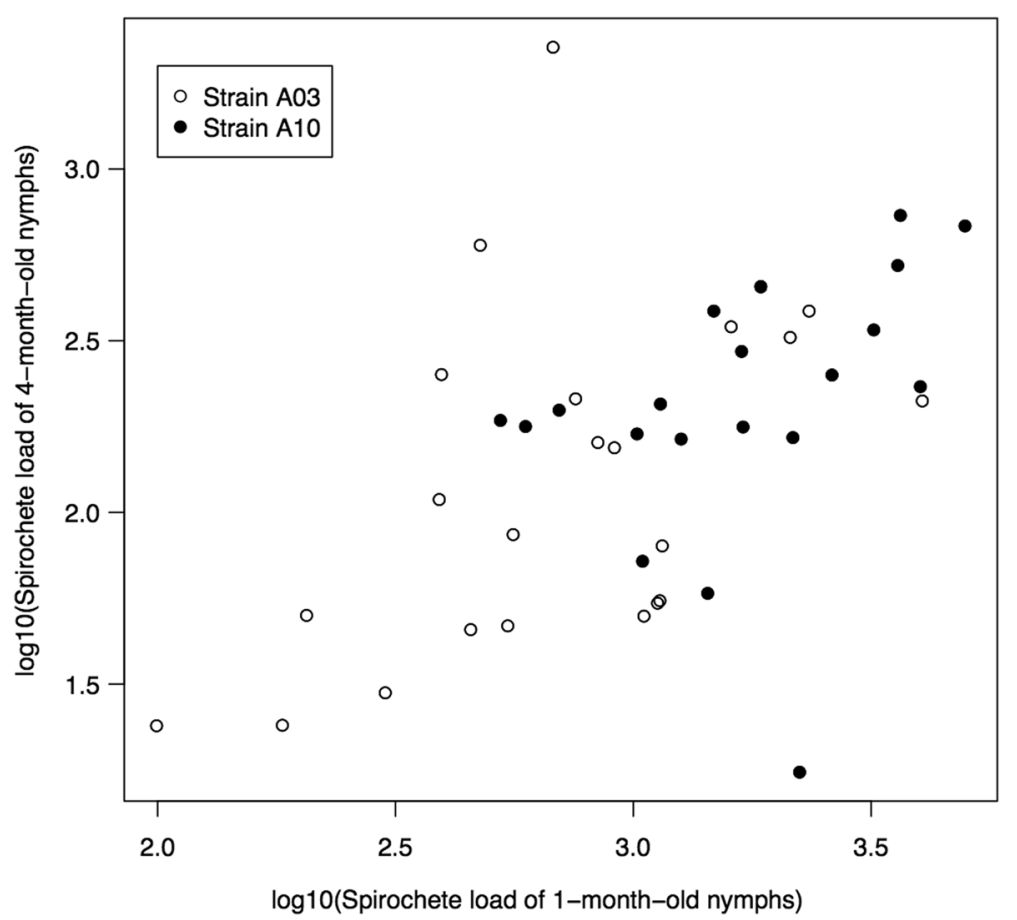

Fig. 2 The log10-transformed nymphal spirochete loads of B. afzelii are highly correlated between the 1-month-old and the 4-month-old I. ricinus nymphs. Strains A3 and A10 are shown with white and black circles, respectively. A3- or A10-infected mice were infested with larval ticks on 43 different occasions. The engorged larval ticks from these infestations were allowed to moult into nymphs and were killed at 1 or 4 months after the larva-to-nymph moult. The spirochete loads of the 1-month-old nymphs and the 4-month-old nymphs therefore represent paired data. Each data point is a mean of the subset of infected nymphs (i.e. uninfected nymphs were excluded) that originated from the same infestation event. The spirochete loads in the nymphs were estimated using a qPCR that targeted a 132 bp fragment of the flagellin gene

\section{Spirochete load of $B$. afzelii in engorged larvae and flat nymphs}

Of the 46 engorged larvae that were randomly sampled, $30.4 \%$ (14/46) tested positive for B. afzelii. The remaining engorged larvae from the same infestation were allowed to moult into nymphs. Of the 86 nymphs that were randomly sampled, 77.9\% (67/86) tested positive for B. afzelii. The proportion of infected ticks increased 2.6-fold from the engorged larvae (tested at $48 \mathrm{~h}$ after drop-off) to the flat nymphs (tested at 4 weeks after the larva-to-nymph moult), and this difference was significant $\left(\chi^{2}=26.52, d f=\right.$ $1, P<0.001)$. The mean spirochete load for the subset of $B$. afzelii-infected engorged larvae $(n=14$, mean: 123, 95\% CI: 53-285 spirochetes per engorged larva) was 20 times smaller than that of the subset of B. afzelii-infected nymphs ( $n=67$, mean: 2416 , 95\% CI: $1646-3546$ spirochetes per nymph) and this difference was significant $(t=$ 6.420, $d f=79, P<0.001$; Fig. 3a).

\section{Spirochete load of B. afzelii in flat nymphs and flat adult ticks}

Of the pool of nymphs used to create the adult ticks, 35 nymphs were randomly selected, and $100.0 \%$ (35/35) tested positive for $B$. afzelii. Of the remaining nymphs, 100 were fed on 20 uninfected mice, and 82 engorged nymphs were recovered, of which 62 moulted into adult ticks. Of these 62 adult ticks, 53.2\% (33/62) tested positive for B. afzelii. The proportion of infected ticks decreased 1.9-fold from the flat nymphs (tested at 4 weeks after the larva-to-nymph moult) to the flat adults (tested at 28 weeks after the nymph-to-adult moult), and this difference was significant $\left(\chi^{2}=21.17, d f=\right.$ $1, P<0.001)$. The percentage of $B$. afzelii-positive ticks was the same between males $(58.1 \%=18 / 31)$ and females $(48.4 \%=15 / 31)$. The mean spirochete load for the subset of $B$. afzelii-infected nymphs ( $n=35$, mean: $5801,95 \%$ CI: 3288-10,234 spirochetes per nymph) was 3 times larger than that for the subset of $B$. afzelii-infected adults $(n=$ 33, mean: 1959, 95\% CI: 1092-3516 spirochetes per adult tick) and this difference was significant $(t=2.659, d f=79, P$ $<0.010$; Fig. $3 \mathrm{~b}$ ). There was no effect of sex on the mean spirochete load in the adult ticks.

\section{Mean spirochete load in wild $I$. ricinus nymphs}

Of the 100 wild $I$. ricinus nymphs that were frozen at 1 month post-molt, 69 were infected with B. afzelii ospC strain A10. The mean spirochete load for the subset of B. afzelii-infected nymphs was 2793 spirochetes per nymph ( $n=69$, 95\% CI: 1761-4492 spirochetes per nymph). 



Fig. 3 The spirochete load of $B$. afzelii in the tick changes over the life-cycle of $I$. ricinus. a The log 10-transformed spirochete load increased 20-fold from the engorged larva (48 h after drop-off) to the nymph (4 weeks after the larva-to-nymph moult). $\mathbf{b}$ The log10-transformed spirochete load decreased 3-fold from the nymph (4 weeks after the larva-to-nymph moult) to the adult (28 weeks after the nymph-to-adult moult). The spirochete loads in the ticks were estimated using a qPCR that targeted a $132 \mathrm{bp}$ fragment of the flagellin gene. The spirochete loads were log10-transformed to improve their fit to the normal distribution. Shown are the medians (black line), the 25th and 75th percentiles (edges of the box), the minimum and maximum values (whiskers), and the outliers (solid circles)

\section{Discussion}

The population of $B$. afzelii spirochetes in $I$. ricinus nymphal ticks declined dramatically over time (Fig. 1). Over a period of 3 months, the mean nymphal spirochete load declined by 82.8 and $88.2 \%$ for strains A3 and A10, respectively. This result was similar to a pilot study on the same system that compared the mean spirochete load between 1-month-old nymphs and 7-month-old nymphs [33]. In that study, the nymphal spirochete load declined by 47.2 and $86.5 \%$ for strains A3 and A10, respectively [33]. The sample size in the present study (432 infected nymphs) is much larger than the pilot study (80 infected nymphs). The paired experimental design used in the present study also provided a more powerful statistical test because it controlled for substantial variation in mean spirochete load between larval infestations (Fig. 2). We are not aware of any other studies on the dynamics of the spirochete population size over time inside unfed nymphal ticks after the larva-tonymph moult. Early microscope studies on B. burgdorferi (s.s.) in I. scapularis quantified the nymphal spirochete load shortly after the larva-to-nymph moult [32] or at a single unspecified time point [26]. The estimates of the nymphal spirochete loads in these microscopebased studies are much lower than the present study. For example, Piesman et al. [32] estimated that I. scapularis nymphs contain $<300$ B. burgdorferi (s.s.) spirochetes per tick at 3 to 12 days after the larva-to-nymph moult. Similarly, De Silva \& Fikrig [26] estimated that nymphs contain 496 spirochetes per tick at some unspecified time point after the larva-to-nymph moult. In contrast, the present study found that I. ricinus nymphs contained a mean of 8880 or 14,094 B. afzelii spirochetes per tick at four weeks after the larva-to-nymph moult depending on the strain. The large differences in nymphal spirochete load between the early studies and the present study might be due to differences in the Borrelia species, the tick species, or the method of quantification (microscopy versus qPCR).

Why does the spirochete population size inside the unfed nymphal tick decline over time? One possible explanation is that digestion of the larval blood meal in the midgut of the unfed nymph reduces the spirochete population over time [18]. Previous work on B. burgdorferi (s.s.) in I. scapularis and on B. burgdorferi (s.l.) in I. ricinus has shown that the spirochete population in the unfed nymph is predominantly located in the tick midgut [26, 39-42]. Molecular studies have identified the outer surface proteins (e.g. OspA and OspB) that Borrelia pathogens use to anchor themselves to the tick midgut [21-24]. Digestion of the blood meal in ticks is a slow process that takes place within the epithelial cells lining the tick midgut $[43,44]$. Endocytosis and intracellular digestion of the blood meal by epithelial cells could also result in the ingestion and destruction of spirochetes [18]. There is indirect evidence suggesting that 
digestion of the larval blood meal continues in unfed nymphs. First, proteomics analysis has detected residual blood proteins from the vertebrate host in unfed nymphs at 3-11 months post-moult [45, 46]. Second, host blood meal studies have shown that the ability to detect and identify host DNA in unfed nymphs decreases over time $[47,48]$. These observations suggest that the digestion of host proteins and host DNA from the larval blood meal continues in unfed nymphs. Thus one explanation for the temporal decline in the spirochete load is the slow but continuous digestion of the larval blood meal and the spirochete population in the unfed nymphal tick.

Does the temporal decline in spirochete load in the nymphal tick influence tick-to-host transmission of the Borrelia pathogen? During the nymphal blood meal, the spirochetes divide in the tick midgut and migrate to the tick salivary glands $[26,41,42]$. This spirochete migration inside the tick explains why the probability of infection increases with the duration of attachment to the host $[5,25,26,30,31]$. A recent study on B. burgdorferi (s.s.) in I. scapularis nymphs found that nymphal age and temperature treatment had no effect on the spirochete load in the engorged nymph [20]. For example, nymphs that were aged for 0 months, 3 months (at $4{ }^{\circ} \mathrm{C}$ ) or 9 months ( 3 months at $4{ }^{\circ} \mathrm{C}$ followed by 6 months at room temperature) all had the same spirochete load following engorgement [20]. This study suggests that spirochete replication following tick attachment might compensate for any temporal decline in nymphal spirochete load. However, other studies suggest that the nymphal spirochete load may influence strain-specific transmission to the vertebrate host. A study on B. afzelii $o s p C$ strains in a wild $I$. ricinus population found that the strains that established the highest spirochete load in the flat nymphs were the most common in the tick population [10]. A study using genetically tagged strains of B. burgdorferi (s.s.) found that the strains with the highest spirochete load in the nymphal tick had a higher probability of being transmitted to the rodent host [8]. These two studies suggest that the spirochete load in the nymphal tick is an important life history trait of Borrelia pathogens. Future studies should investigate whether the temporal decline in the nymphal spirochete load influences the tick-to-host transmission success of Borrelia pathogens.

Does the temporal decline in spirochete load influence our ability to detect Borrelia infection in nymphal ticks? In the first experiment, the proportion of infected ticks did not change between the 1-month-old nymphs and the 4-month-old nymphs. This result demonstrates that the sensitivity of our qPCR assay is high and that the probability to detect $B$. afzelii infection in $I$. ricinus nymphs was not affected by a seven-fold drop in spirochete load. In contrast, a study on B. burgdorferi (s.s.) in
I. scapularis nymphs using conventional PCR found that the proportion of infected ticks declined from 74.0 to $15.5 \%$ between 1-month-old nymphs and 5-month-old nymphs [49]. One interpretation of these data is that the conventional PCR was unable to detect the infection once the spirochete load dropped below a certain threshold. A limitation with PCR-based methods is that we cannot distinguish between spirochetes that are dead or alive. Future investigations should use methods that allow us to assess microbial viability [50].

The present study showed that the population of $B$. afzelii spirochetes increased dramatically from the engorged larval tick to the flat nymphal tick (Fig. 3a). A larval tick acquired about 100 spirochetes after feeding on an infected mouse, and this spirochete population grew exponentially so that it was 20 times larger in the nymph at 4 weeks after the larva-to-nymph moult. The early microscope study on B. burgdorferi (s.s.) in I. scapularis by Piesman et al. [32] showed a similarly dramatic expansion of the spirochete population inside the engorged larval tick over the first 15 days post-repletion. The study by Piesman et al. [32] did not estimate the number of spirochetes acquired by a feeding larval tick, but extrapolation of their data suggests that the inoculum size is $<250$ spirochetes. The observation in the present study that the proportion of infected ticks increased 2.6-fold from the engorged larval ticks to the flat nymphs is an issue of detection and false negatives. In some engorged larval ticks, the number of spirochetes acquired was so low that our qPCR assay did not detect them. Another explanation for the low ability to detect B. afzelii infections in engorged larvae is that heme in blood is a known inhibitor of PCR [51, 52]. The larval blood meal is an important bottleneck that determines the genetic diversity of the Borrelia population that is acquired by the larval tick and subsequently transmitted by the resultant nymph [8]. Thus accurate estimates of how many spirochetes are acquired by a larval tick are important for understanding the population genetics of Borrelia spirochetes [53].

Our study found that the spirochete load in the unfed adult ticks was three times lower than that in the unfed nymphal ticks (Fig. 3b). Numerous studies have shown that the spirochete load inside the nymph increases dramatically during the nymphal blood meal $[20,26-28,32$, $33,54,55]$. For example, the mean spirochete load of 7 month-old flat nymphs infected with $B$. afzelii ospC strains A3 and A10 (743 and 1537 spirochetes), increased by a factor of 4.8 and 1.9, respectively, when the nymphs engorged on the laboratory mice (3530 and 2896 spirochetes) 4 months later [33]. In those studies, the engorged nymphs were killed during or shortly after completing the blood meal. In the present study, by contrast, the adult ticks were killed at 198 days after the 
nymph-to-adult moult. If adult ticks lose spirochetes at the same rate as observed for nymphs in the present study (82-208 spirochetes per day), we would expect some of the spirochete populations to go extinct after 198 days of blood meal digestion. Thus, one explanation for the observation that the prevalence of $B$. afzelii declined from $100.0 \%$ in the flat nymphs to $53.2 \%$ in the flat adults was that the spirochete population had decreased below the qPCR detection threshold. Life history theory suggests that selection is weak for Borrelia pathogens to maintain a viable spirochete population in adult ticks. Adult ticks are dead-end vectors for Borrelia because the males do not blood feed and because the females tend to feed on incompetent reservoir hosts such as deer [56-58]. We therefore expect Borrelia spirochete populations to decline in quantity and quality over time inside adult ticks. Future studies should investigate whether the observed decline in spirochete load in the nymphal ticks also occurs in the adult ticks.

Is the spirochete load of $B$. afzelii in our laboratory population of $I$. ricinus ticks similar to what is observed in wild ticks? Our laboratory population of I. ricinus was domesticated at the University of Neuchâtel almost 40 years ago [59]. It is possible that laboratory selection has changed the susceptibility of our laboratory population of $I$. ricinus to infection with $B$. afzelii. We therefore collected wild $I$. ricinus larva at a field site near Neuchâtel, fed them on lab mice infected with $B$. afzelii isolate NE4049 (ospC strain A10), and tested the nymphs at 1 month post-moult. The prevalence of $B$. afzelii infection was 1.4 times higher for the lab nymphs than the wild nymphs. This observation suggests that lab ticks are more susceptible to acquire B. afzelii infection than wild nymphs. The mean spirochete load in these 1-monthold wild nymphs was 8.2 times lower than the 1-monthold lab nymphs but similar to that of the 4-month-old lab nymphs. This observation suggests that the laboratory ticks are less capable of controlling the B. afzelii spirochete load than the wild nymphs.

In addition, we recently quantified the Borrelia spirochete load in field-collected unfed I. ricinus nymphs [60]. Most of these nymphs were collected in the spring and would have fed as larvae the previous summer or fall, suggesting that the Borrelia infections were 6 to 9 months old. For the 788 wild I. ricinus nymphs that were infected with B. afzelii, the geometric mean spirochete load (2003 spirochetes per nymph) [60] was similar to that observed for the 4-month-old nymphs (1999 spirochetes per nymph; averaged across the two strains) in the present study. Taken together, these studies suggest that the high $B$. afzelii spirochete loads observed in the 1month-old lab nymphs may be a phenotype that is specific to our laboratory population of I. ricinus. Future studies should investigate whether the observed declines in Borrelia spirochete load occur in wild ticks under natural conditions.

\section{Conclusions}

In the present study, we showed that the abundance of B. afzelii in unfed I. ricinus nymphs declines by as much as $90 \%$ over a period of 3 months when nymphs were kept under laboratory conditions. Researchers interested in quantifying the spirochete load in ticks should be aware that this phenotype declines dramatically over time. Future studies should investigate whether the Borrelia spirochete load declines over time in wild Ixodes ticks under natural conditions and whether these changes in spirochete load influence the tick-to-host transmission success of Borrelia pathogens.

\section{Additional file}

Additional file 1: Table S1. List of definitions for the variables that appear in the column headings of Tables S2, S3 and S4. Table S2. Raw data of the Borrelia afzelii spirochete loads for the 1-month-old Ixodes ricinus nymphs and the 4-month-old I. ricinus nymphs used to create Figs. 1 and 2. Table S3. Raw data of the B. afzelii spirochete loads for the engorged larval ticks and the resultant flat nymphs used to create Fig. $3 a$ and the raw data of the $B$. afzelii spirochete loads for the flat nymphs and the resultant adult ticks used to create Fig. 3b. Table S4. Raw data of the B. afzelii spirochete loads for the wild I. ricinus nymphs. (XLSX 96 kb)

\section{Abbreviations}

ELISA: Enzyme-linked immunosorbent assay; LB: Lyme borreliosis;

OspC: Outer surface protein C; qPCR: Quantitative real-time polymerase chain reaction.

\section{Acknowledgements}

This study is part of the PhD thesis of Maxime Jacquet

Funding

This work was supported by an SNSF grant to MJV (FN 31003A_141153).

Availability of data and materials

The datasets generated and analysed in the present study are available in Additional file 1.

\section{Authors' contributions}

MJV and MJ conceived and designed the study. MJ conducted the experimental work on the laboratory I. ricinus ticks. DG created the engorged larval ticks and the resultant nymphs. $A B$ created the nymphal ticks and the resultant adults. EM and AS conducted the experimental work on the wild I. ricinus ticks. MJ conducted the statistical analyses. MJ and MJV wrote the manuscript. All authors read and approved the final manuscript.

\section{Competing interests}

The authors declare that they have no competing interests.

\section{Consent for publication}

Not applicable.

\section{Ethics approval and consent to participate}

The commission that is part of the 'Service de la Consommation et des Affaires Vétérinaires (SCAV)' of Canton Vaud, Switzerland evaluated and approved the ethics of this study. The Veterinary Service of the Canton of Neuchâtel, Switzerland issued the animal experimentation permits used in this study (NE2/2012 and NE4/2014). 


\section{Publisher's Note}

Springer Nature remains neutral with regard to jurisdictional claims in published maps and institutional affiliations.

Received: 9 December 2016 Accepted: 11 May 2017

Published online: 25 May 2017

\section{References}

1. Rosenberg R, Wirtz RA, Schneider I, Burge R. An estimation of the number of malaria sporozoites ejected by a feeding mosquito. Trans $\mathrm{R}$ Soc Trop Med Hyg. 1990;84(2):209-12.

2. Watts DM, Burke DS, Harrison BA, Whitmire RE, Nisalak A. Effect of temperature on the vector efficiency of Aedes aegypti for dengue 2 virus. Am J Trop Med Hyg. 1987;36(1):143-52.

3. Lima CMR, Zeidner NS, Beard CB, Soares CAG, Dolan MC, Dietrich G, et al. Differential infectivity of the Lyme disease spirochete Borrelia burgdorferi derived from Ixodes scapularis salivary glands and midgut. J Med Entomol. 2005:42(3):506-10.

4. Jin Y, Kebaier C, Vanderberg J. Direct microscopic quantification of dynamics of Plasmodium berghei sporozoite transmission from mosquitoes to mice. Infect Immun. 2007;75(11):5532-9.

5. Cook MJ. Lyme borreliosis: a review of data on transmission time after tick attachment. Int J Gen Med. 2015:8:1-8.

6. Richards SL, Anderson SL, Lord CC, Tabachnick WJ. Effects of virus dose and extrinsic incubation temperature on vector competence of Culex nigripalpus (Diptera: Culicidae) for St. Louis encephalitis virus. J Med Entomol. 2012; 49(6):1502-6.

7. Lambrechts L, Paaijmans KP, Fansiri T, Carrington LB, Kramer LD, Thomas $M B$, et al. Impact of daily temperature fluctuations on dengue virus transmission by Aedes aegypti. Proc Natl Acad Sci USA. 2011;108(18):7460-5.

8. Rego ROM, Bestor A, Stefka J, Rosa PA. Population bottlenecks during the infectious cycle of the Lyme disease spirochete Borrelia burgdorferi. PLOS One. 2014;9(6):e101009

9. Reif KE, Palmer GH, Crowder DW, Ueti MW, Noh SM. Restriction of Francisella novicida genetic diversity during infection of the vector midgut. PLOS Pathog. 2014;10(11):1-11.

10. Durand J, Herrmann C, Genné D, Sarr A, Gern L, Voordouw MJ. Multistrain infections with Lyme borreliosis pathogens in the tick vector. Appl Environ Microbiol. 2017;83(3).

11. Parham PE, Waldock J, Christophides GK, Hemming D, Agusto F, Evans KJ, et al. Climate, environmental and socio-economic change: weighing up the balance in vector-borne disease transmission. Philos T Roy Soc B. 2015;370(1665):1-17

12. Kurtenbach K, Hanincova K, Tsao Jl, Margos G, Fish D, Ogden NH. Fundamental processes in the evolutionary ecology of Lyme borreliosis. Nat Rev Microbiol. 2006;4:660-9.

13. Piesman J, Gern L. Lyme borreliosis in Europe and North America. Parasitology. 2004;129:S191-220.

14. Schotthoefer AM, Frost HM. Ecology and epidemiology of Lyme borreliosis. Clin Lab Med. 2015;35(4):723-43.

15. Lindsay LR, Barker IK, Surgeoner GA, McEwen SA, Campbell GD. Duration of Borrelia burgdorferi infectivity in white-footed mice for the tick vector Ixodes scapularis under laboratory and field conditions in Ontario. J Wildl Dis. 1997;33(4):766-75

16. Randolph SE. Ticks are not insects: consequences of contrasting vector biology for transmission potential. Parasitol Today. 1998;14(5):186-92.

17. Talleklint $L$, Jaenson TGT. Is the small mammal (Clethrionomys glareolus) or the tick vector (Ixodes ricinus) the primary overwintering reservoir for the Lyme borreliosis spirochete in Sweden. J Wildl Dis. 1995:31(4):537-40.

18. Kung F, Anguita J, Pal U. Borrelia burgdorferi and tick proteins supporting pathogen persistence in the vector. Future Microbiol. 2013;8(1):41-56.

19. Drecktrah D, Lybecker M, Popitsch N, Rescheneder P, Hall LS, Samuels DS. The Borrelia burgdorferi ReIA/SpoT homolog and stringent response regulate survival in the tick vector and global gene expression during starvation. PLOS Pathog. 2015:11(9):1-39.

20. Fazzino L, Tilly K, Dulebohn DP, Rosa PA. Long term survival of Borrelia burgdorferi lacking hibernation promotion factor homolog in the unfed tick vector. Infect Immun. 2015;83(12):4800-10.
21. Neelakanta G, Li X, Pal U, Liu X, Beck DS, DePonte K, et al. Outer surface protein $\mathrm{B}$ is critical for Borrelia burgdorferi adherence and survival within Ixodes ticks. PLOS Pathog. 2007;3(3):1-11.

22. Pal U, de Silva AM, Montgomery RR, Fish D, Anguita J, Anderson JF, et al. Attachment of Borrelia burgdorferi within Ixodes scapularis mediated by outer surface protein A. J Clin Invest. 2000;106(4):561-9.

23. Pal U, Li X, Wang T, Montgomery RR, Ramamoorthi N. deSilva AM, et al. TROSPA, an Ixodes scapularis receptor for Borrelia burgdorferi. Cell. 2004;119(4):457-68.

24. Yang XFF, Pal U, Alani SM, Fikrig E, Norgard MV. Essential role for OspA/B in the life cycle of the Lyme disease spirochete. J Exp Med. 2004;199(5):641-8.

25. Piesman J, Mather TN, Sinsky RJ, Spielman A. Duration of tick attachment and Borrelia burgdorferi transmission. J Clin Microbiol. 1987;25(3):557-8.

26. de Silva AM, Fikrig E. Growth and migration of Borrelia burgdorferi in Ixodes ticks during blood feeding. Am J Trop Med Hyg. 1995;53(4):397-404.

27. Ohnishi J, Piesman J, de Silva AM. Antigenic and genetic heterogeneity of Borrelia burgdorferi populations transmitted by ticks. Proc Natl Acad Sci USA 2001;98(2):670-5.

28. Piesman J, Schneider BS, Zeidner NS. Use of quantitative PCR to measure density of Borrelia burgdorferi in the midgut and salivary glands of feeding tick vectors. J Clin Microbiol. 2001:39(11):4145-8.

29. Piesman J. Dynamics of Borrelia burgdorferi transmission by nymphal /xodes dammini ticks. J Infect Dis. 1993:167(5):1082-5.

30. Kahl O, Janetzki-Mittmann C, Gray JS, Jonas R, Stein J, de Boer R. Risk of infection with Borrelia burgdorferi sensu lato for a host in relation to the duration of nymphal Ixodes ricinus feeding and the method of tick removal. Zbl Bakt-Int J Med M. 1998;287(1-2):41-52.

31. Crippa M, Rais O, Gern L. Investigations on the mode and dynamics of transmission and infectivity of Borrelia burgdorferi sensu stricto and Borrelia afzelii in Ixodes ricinus ticks. Vector Borne and Zoonotic Diseases. 2002;2(1):3-9.

32. Piesman J, Oliver JR, Sinsky RJ. Growth kinetics of the Lyme disease spirochete (Borrelia burgdorferi) in vector ticks (Ixodes dammini). Am J Trop Med Hyg. 1990:42(4):352-7.

33. Jacquet M, Durand J, Rais O, Voordouw MJ. Cross-reactive acquired immunity influences transmission success of the Lyme disease pathogen, Borrelia afzelii. Infect Genet Evol. 2015;36:131-40.

34. Jacquet M, Durand J, Rais O, Voordouw MJ. Strain-specific antibodies reduce co-feeding transmission of the Lyme disease pathogen, Borrelia afzelii. Environ Microbiol. 2016;18(3):833-45.

35. Tonetti N, Voordouw MJ, Durand J, Monnier S, Gern L. Genetic variation in transmission success of the Lyme borreliosis pathogen Borrelia afzelii. Ticks Tick Borne Dis. 2015:6(3):334-43.

36. Jacquet M, Margos G, Fingerle V, Voordouw MJ. Comparison of the lifetime host-to-tick transmission between two strains of the Lyme disease pathogen Borrelia afzelii. Parasit Vectors. 2016;9:1-8.

37. Schwaiger $M$, Peter $O$, Cassinotti P. Routine diagnosis of Borrelia burgdorferi (sensu lato) infections using a real-time PCR assay. Clin Microbiol Infec. 2001;7(9):461-9.

38. R Development Core Team: R. A language and environment for statistical computing. Vienna: R Foundation for Statistical Computing; 2009.

39. Burgdorfer W, Anderson JF, Gern L, Lane RS, Piesman J, Spielman A Relationship of Borrelia burgdorferi to its arthropod vectors. Scand J Infect Dis. 1991;S77:35-40.

40. Burgdorfer W, Hayes SF, Corwin D. Patho-physiology of the Lyme disease spirochete, Borrelia burgdorferi, in ixodid ticks. Rev Infect Dis. 1989;11:S1442-S50.

41. Dunham-Ems SM, Caimano MJ, Pal U, Wolgemuth CW, Eggers CH, Balic A et al. Live imaging reveals a biphasic mode of dissemination of Borrelia burgdorferi within ticks. J Clin Invest. 2009;119(12):3652-65.

42. Gern L, Lebet N, Moret J. Dynamics of Borrelia burgdorferi infection in nymphal Ixodes ricinus ticks during feeding. Exp Appl Acarol. 1996;20(11):649-58.

43. Sojka D, Franta Z, Horn M, Caffrey CR, Mares M, Kopacek P. New insights into the machinery of blood digestion by ticks. Trends Parasitol. 2013:29(6):276-85.

44. Sonenshine DE. Biology of Ticks, vol. 1. New York: Oxford University Press; 1993.

45. Wickramasekara S, Bunikis J, Wysocki V, Barbour AG. Identification of residual blood proteins in ticks by mass spectrometry proteomics. Emerg Infect Dis. 2008;14(8):1273-5. 
46. Laskay UA, Breci L, Vilcins IME, Dietrich G, Barbour AG, Piesman J, et al. Survival of host blood proteins in Ixodes scapularis (Acari: Ixodidae) ticks: a time course study. J Med Entomol. 2013;50(6):1282-90.

47. Morán Cadenas FM, Rais $\mathrm{O}$, Humair PF, Douet V, Moret J, Gern L. Identification of host bloodmeal source and Borrelia burgdorferi sensu lato in field-collected Ixodes ricinus ticks in Chaumont (Switzerland). J Med Entomol. 2007;44(6):1109-17.

48. Pichon B, Egan D, Rogers M, Gray J. Detection and identification of pathogens and host DNA in unfed host-seeking lxodes ricinus L. (Acari: |xodidae). J Med Entomol. 2003;40(5):723-31.

49. Voordouw MJ, Tupper H, Oender O, Devevey G, Graves CJ, Kemps BD, et al. Reductions in human Lyme disease risk due to the effects of oral vaccination on tick-to-mouse and mouse-to-tick transmission. Vector-Borne Zoonot. 2013;13(4):203-14.

50. Cangelosi GA, Meschke JS. Dead or alive: molecular assessment of microbial viability. Appl Environ Microbiol. 2014;80(19):5884-91.

51. Buckwalter SP, Sloan LM, Cunningham SA, Espy MJ, Uhl JR, Jones MF, et al. Inhibition controls for qualitative real-time PCR assays: are they necessary for all specimen matrices? J Clin Microbiol. 2014;52(6):2139-43.

52. Schrader C, Schielke A, Ellerbroek L, Johne R. PCR inhibitors - occurrence, properties and removal. J Appl Microbiol. 2012;113(5):1014-26.

53. Seifert SN, Khatchikian CE, Zhou W, Brisson D. Evolution and population genomics of the Lyme borreliosis pathogen. Borrelia burgdorferi Trends Genet. 2015;31(4):201-7.

54. Piesman J, Schneider BS. Dynamic changes in Lyme disease spirochetes during transmission by nymphal ticks. Exp Appl Acarol. 2002:28(1):141-5.

55. Zhu Z. Histological observations on Borrelia burgdorferi growth in naturally infected female Ixodes ricinus. Acarologia. 1998;39(1):11-22.

56. Jaenson TGT, Talleklint L. Incompetence of roe deer as reservoirs of the Lyme borreliosis spirochete. J Med Entomol. 1992;29(5):813-7.

57. Telford SR, Mather TN, Moore SI, Wilson ML, Spielman A. Incompetence of deer as reservoirs of the Lyme-disease spirochete. Am J Trop Med Hyg. 1988;39(1):105-9.

58. Matuschka FR, Fischer P, Heiler M, Richter D, Spielman A. Capacity of European animals as reservoir hosts for the Lyme disease spirochete. J Infect Dis. 1992;165(3):479-83.

59. Graf J-F. Copulation, nutrition et ponte chez Ixodes ricinus L. (Ixodoidea: Ixodidae) - 1e partie. Bulletin de la Société Entomologique Suisse. 1978:51:89-97.

60. Herrmann C, Gern L, Voordouw MJ. Species co-occurrence patterns among Lyme borreliosis pathogens in the tick vector Ixodes ricinus. Appl Environ Microbiol. 2013;79(23):7273-80

\section{Submit your next manuscript to BioMed Central and we will help you at every step:}

- We accept pre-submission inquiries

- Our selector tool helps you to find the most relevant journal

- We provide round the clock customer support

- Convenient online submission

- Thorough peer review

- Inclusion in PubMed and all major indexing services

- Maximum visibility for your research

Submit your manuscript at www.biomedcentral.com/submit

) Biomed Central 